\title{
Metformin reduces chondrocyte pyroptosis in an osteoarthritis mouse model by inhibiting NLRP3 inflammasome activation
}

\author{
JIANGBO YAN $^{1 *}$, DONG DING $^{1 *}$, GANGNING FENG $^{1}$, YONG YANG $^{1,2}$, YONG ZHOU $^{1}$, \\ LONG MA ${ }^{2}$, HAOHUI GUO ${ }^{2}$, ZHIDONG LU ${ }^{2}$ and QUNHUA JIN ${ }^{2}$ \\ ${ }^{1}$ Clinical Medical School, Ningxia Medical University; ${ }^{2}$ Orthopedics Ward 3, The General Hospital of \\ Ningxia Medical University, Yinchuan, Ningxia Hui Autonomous Region 750004, P.R. China
}

Received October 25, 2021; Accepted December 21, 2021

DOI: $10.3892 /$ etm.2022.11146

\begin{abstract}
Osteoarthritis (OA) is an age-related degenerative disease, and its incidence is increasing with the ageing of the population. Metformin, as the first-line medication for the treatment of diabetes, has received increasing attention for its role in OA. The purpose of the present study was to confirm the therapeutic effect of metformin in a mouse model of OA and to determine the mechanism underlying the resultant delay in OA progression. The right knees of 8-week-old C57BL/6 male mice were subjected to destabilization of the medial meniscus (DMM). Metformin (200 mg/kg) was then administered daily for 4 or 8 weeks. Safranin O-fast green staining, H\&E staining and micro-CT were used to analyse the structure and morphological changes. Immunohistochemical staining was used to detect type II collagen (Col II), matrix metalloproteinase 13 (MMP-13), NOD-like receptor protein 3 (NLRP3), caspase-1, gasdermin D (GSDMD) and IL-1 $\beta$ protein expression. Reverse transcription-quantitative PCR was used to detect the
\end{abstract}

Correspondence to: Professor Zhidong Lu or Professor Qunhua Jin, Orthopedics Ward 3, The General Hospital of Ningxia Medical University, 804 Shengli South Street, Yinchuan, Ningxia Hui Autonomous Region 750004, P.R. China

E-mail: zhidonglu@nyfy.com.cn

E-mail: jinqunhua2020@163.com

*Contributed equally

Abbreviations: OA, osteoarthritis; DMM, destabilization of the medial meniscus; MMP, matrix metalloproteinase; Col II, type II collagen; NLRP3, NOD-like receptor protein 3; ASC, apoptosis-associated speck-like protein containing CARD; GSDMD, gasdermin D; AMPK, adenosine 5'-monophosphate-activated protein kinase; PBS, phosphate-buffered saline; OARSI, Osteoarthritis Research Society International; HC, hyaline cartilage; CC, calcified cartilage; RT-qPCR, reverse transcription-quantitative PCR; BV/TV, bone volume fraction; Tb.Sp, trabecular separation; $\mathrm{BMD}$, bone mineral density

Key words: osteoarthritis, chondrocytes, metformin, pyroptosis, NOD-like receptor protein 3
mRNA expression of NLRP3, caspase-1, GSDMD and IL-1 $\beta$. Histomorphological staining showed that metformin delayed the progression of OA in the DMM model. With respect to cartilage, metformin decreased the Osteoarthritis Research Society International score, increased the thickness of hyaline cartilage and decreased the thickness of calcified cartilage. Regarding the mechanism, in cartilage, metformin increased the expression of Col II and decreased the expression of MMP-13, NLRP3, caspase-1, GSDMD and IL-1 $\beta$. In addition, in subchondral bone, metformin inhibited osteophyte formation, increased the bone volume fraction (\%) and the bone mineral density $\left(\mathrm{g} / \mathrm{cm}^{3}\right)$, decreased the trabecular separation $(\mathrm{mm})$ in early stage of osteoarthritis (4 weeks) but the opposite in an advanced stage of osteoarthritis (8 weeks). Overall, metformin inhibited the activation of NLRP3 inflammasome, decreased cartilage degradation, reversed subchondral bone remodelling and inhibited chondrocyte pyroptosis.

\section{Introduction}

Osteoarthritis (OA) is an age-related degenerative joint disease that is characterized by the loss of articular cartilage, synovitis, subchondral bone sclerosis and osteophyte formation (1). With the intensification of population ageing, the incidence of OA has increased from 29.2 to $40.5 / 1,000$ person-years between 1992 and 2013 (2), and OA seriously endangers the health of elderly people (3). At present, the aetiology and pathogenesis of OA are still not completely clear; OA is generally considered the result of the joint contribution of biological and mechanical factors (4). The death of cartilage cells, the only type of cell in articular cartilage, is considered the main biological factor (5). A large amount of extracellular matrix, known as type II collagen (Col II), surrounds chondrocytes (6). Matrix metalloproteinase (MMP)-3 and MMP-13 are two main enzymes responsible for the degradation of cartilage matrix (7). Decreased anabolism of cartilage extracellular matrix components, such as Col II and proteoglycans, and increased cartilage-related catabolism components, such as MMP-13 and MMP-3, are characteristic manifestations of cartilage cell degradation (8). Therefore, delaying the degradation and death of chondrocytes is of major significance for delaying the pathological process of OA (5). 
Pyroptosis is a newly discovered mechanism of cell death; it is characterized by cell swelling and rupture, cell membrane dissolution, release of cytoplasmic contents to the outside of the cell and chromosomal DNA breakage (9). The typical activation pathway of pyroptosis is mediated by the NOD-like receptor protein 3 (NLRP3) inflammasome, which mainly includes NLRP3, apoptosis-associated speck-like protein containing CARD (ASC) and pro-caspase-1 (10). In the typical activation pathway, NLRP3 is activated in response to stimuli inside and outside the cell, recruits ASC and assembles into the NLRP3 inflammasome by combining ASC with pro-caspase-1 (11). The activated NLRP3 inflammasome cleaves pro-caspase- 1 into activated caspase-1, which can both cleave the precursors of IL-1 $\beta$ and IL-18, and promote their maturation and secretion. The inflammasome can also cleave gasdermin D (GSDMD) and create GSDMD-N pores in the membrane, thereby inducing cell pyroptosis (12).

There is evidence that the NLRP3 inflammasome is involved in the pathogenesis of OA and can lead to cartilage degeneration and synovial inflammation through the activation of Toll-like receptors and NF- $\kappa \mathrm{B}$ signal transduction (13). The latest research shows that exogenous stromal cell-derived factor-1 inhibits the NLRP3 inflammasome by activating the adenosine 5'-monophosphate-activated protein kinase (AMPK) signalling pathway, and that it inhibits the pyroptosis of synovial cells in OA (14). The NLRP3 inflammasome has gradually become a new therapeutic target for OA (15). The pathways that activate the NLRP3 inflammasome, such as potassium efflux and the production of reactive oxygen species, have been extensively studied (16). Currently, the literature on the involvement of the NLRP3 inflammasome in the pathogenesis of $\mathrm{OA}$ and its potential use as a biomarker is limited.

Metformin is the first-line medication for diabetes treatment (17). Studies have shown that metformin, as an AMPK activator, can affect bone metabolism (18) and enhance the anti-inflammatory properties of experimental OA mesenchymal stem cells (19). Moreover, it has been indicated that metformin can reverse the interferon-inducible protein AIM2-related pyroptosis of macrophages caused by diabetes (20), and can protect against myocardial ischaemia-reperfusion injury and decrease myocardial cell pyroptosis through the AMPK/NLRP3 inflammasome pathway (21). Metformin can also exert an anti-periodontitis effect by targeting the NLRP3 inflammasome (22). However, the mechanism through which metformin affects the activation of NLRP3 inflammasome in OA chondrocytes and delays the progression of OA remains unclear.

To the best of the authors' knowledge, the effect of metformin on the pyroptosis of OA chondrocytes remains to be elucidated. In the present study, an OA mouse model was constructed through destabilization of the medial meniscus (DMM) surgery to investigate the therapeutic effects of metformin on knee OA in mice from a novel perspective.

\section{Materials and methods}

Ethics statement. All experiments were approved by the Animal Experiment Ethics Committee of Ningxia Medical University (Yinchuan, China; protocol no. 2020-115). All experiments were conducted under the standard principles of animal experiment ethics.

Animals and the DMM-induced OA model. All animals in the present study were healthy, wild-type, adult male C57BL/6 mice [specific pathogen-free (SPF) grade], aged 8 weeks and weighing 20-25 g. The mice were obtained from the Laboratory Animal Centre of Ningxia Medical University. All experimental subjects were maintained in an SPF environment at a temperature of $22 \pm 1^{\circ} \mathrm{C}$ with a humidity of $55 \%$, a 12-h light/dark cycle and free access to food and water. In the experiment, surgically induced OA was achieved through DMM. First, the mice were weighed and anesthetized via intraperitoneal injection of $0.2 \%$ pentobarbital sodium $(40 \mathrm{mg} / \mathrm{kg})$ in phosphate-buffered saline (PBS; OriGene Technologies, Inc.). After cutting off the nodular sac, the meniscus tibial ligament was cut open by microsurgery, and the anterior horn of the medial meniscus was freed to ensure that the tibial ligament of the inner meniscus was cut. The procedures for the sham operation were the same as those for the DMM operation except that the joint capsule was sutured directly after opening and confirming the absence of abnormalities; that is, no treatment was performed on the meniscus tibial ligament.

Experimental design and processing. A total of 48 mice were randomly divided into three groups (16 in each group) as follows: A control group (sham operation group), an OA group (DMM group) and a metformin (Sino-American Shanghai Squibb Pharmaceuticals Ltd.) treatment group. The metformin treatment group was administered daily metformin (200 mg/kg) via oral gavage based on the results of a previous study (23), with administration initiated on the second day after surgery ( 8 mice for 4 weeks and another 8 mice for 8 weeks). The sham operation and DMM groups were administered an equivalent dose of normal saline as a drug-treatment control. At 4 and 8 weeks following surgery, 8 mice from each group were sacrificed by cervical dislocation, and knee joint tissues were collected to evaluate the severity of OA. The schematic diagram of the animal experiments is presented in Fig. 1.

Histological evaluation of articular cartilage degeneration. Follow-up experiments were performed on the right knee joints of 5 mice in each group. The right knee joint of each mouse was fixed in $4 \%$ paraformaldehyde for $24 \mathrm{~h}$ at $20^{\circ} \mathrm{C}$, rinsed with PBS every hour for $6 \mathrm{~h}$, and then decalcified with 10\% EDTA (cat. no. AR1071; Wuhan Boster Biological Technology Co., Ltd.) for 2 weeks at $20^{\circ} \mathrm{C}$. Next, the tissue was dehydrated with gradient ethanol and embedded in paraffin. The thickness of the sagittal section of the knee joint was $4 \mu \mathrm{m}$, and the sections were stained with safranin $\mathrm{O}$-fast green and H\&E. According to H\&E staining kit (cat. no. G1005; Wuhan Servicebio Technology Co., Ltd.), the sections were placed in xylene twice for $15 \mathrm{~min}$, anhydrous ethanol twice (soaked for $7 \mathrm{~min}$ ) and finally, in $75 \%$ alcohol for $7 \mathrm{~min}$. The sections were stained with hematoxylin at $20^{\circ} \mathrm{C}$ for $5 \mathrm{~min}$, rinsed in running water for $5 \mathrm{~min}$, dehydrated in 85 and $95 \%$ alcohol for $10 \mathrm{~min}$ each, and finally stained with eosin at $20^{\circ} \mathrm{C}$ for $5 \mathrm{~min}$. For staining with safranin $\mathrm{O}$-fast green (cat. no. G1053; Wuhan Servicebio Technology Co., Ltd.), the deparaffinization of slides were consistent with the description 

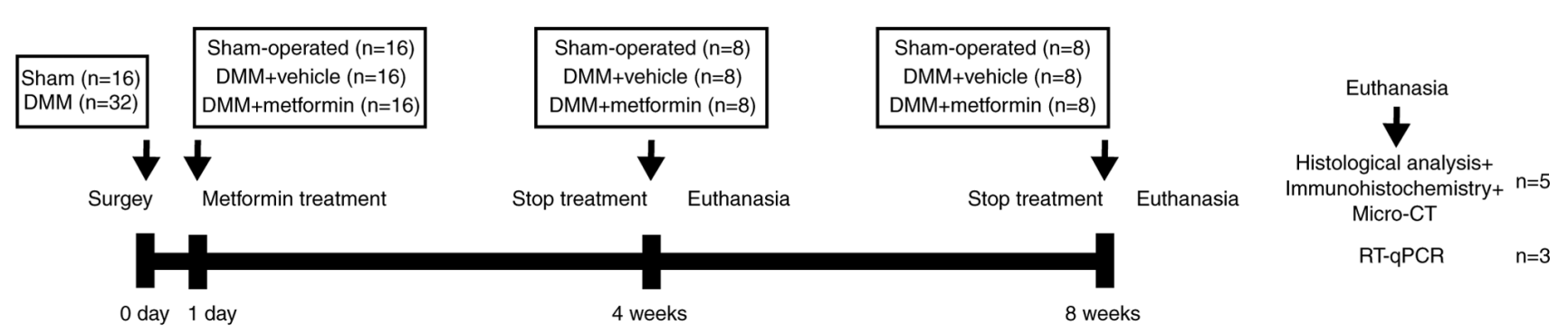

Figure 1. Schematic diagram of the animal experiment. DMM, destabilization of the medial meniscus; RT-qPCR, reverse transcription-quantitative PCR.

above, and subsequently stained with Fast Green for 6 min, washed at $20^{\circ} \mathrm{C}$, dehydrated and stained with Safranin O at $20^{\circ} \mathrm{C}$ for $3 \mathrm{~min}$. Using the Osteoarthritis Research Society International (OARSI) scoring system to evaluate the degeneration of articular cartilage, as described previously (24). The distance from the tidemark to the surface of the articular cartilage was measured and recorded as the thickness of the hyaline cartilage (HC), and the distance from the tidemark to the subchondral bone plate was recorded as the thickness of the calcified cartilage (CC). Then, five random views from three sections per mouse were visualized using a DP71 light microscope with DP controller 3.3.1.292 software (Olympus Corporation) and quantified using ImageJ $1.48 \mathrm{v}$ software (National Institutes of Health).

Immunohistochemistry. Paraffin-embedded mouse knee joint sections at a thickness of $4 \mu \mathrm{m}$ were deparaffinized with xylene and rehydrated with a graduated ethanol series. For antigen retrieval, $0.1 \%$ trypsin was applied to each section at $37^{\circ} \mathrm{C}$ for $15 \mathrm{~min}$, and the sections were incubated with $3 \%$ hydrogen peroxide at $37^{\circ} \mathrm{C}$ for $10 \mathrm{~min}$ to deactivate endogenous peroxidase. Next, the sections were blocked with $5 \%$ normal goat serum (OriGene Technologies, Inc.) at $37^{\circ} \mathrm{C}$ for $30 \mathrm{~min}$ and incubated overnight at $4^{\circ} \mathrm{C}$ with the following primary antibodies: Anti-MMP-13 (cat. no. ab39012; 1:300 dilution; Abcam), anti-Col II (cat. no. ab34712; 1:300 dilution; Abcam), anti-NLRP3 (cat. no. ab214185; 1:200 dilution; Abcam), anti-caspase-1 (cat. no. 22915-1-AP; 1:200 dilution; ProteinTech Group, Inc.), anti-GSDMD (cat. no. ab219800; 1:200 dilution; Abcam) and anti-IL-1 $\beta$ (cat. no. ab205924; 1:300 dilution; Abcam). After that, sections were processed using a two-step IHC detection reagent (ZSGB-Bio; OriGene Technologies, Inc.). Briefly, sections were incubated with reaction enhancement solution (reagent 1) at $37^{\circ} \mathrm{C}$ for $30 \mathrm{~min}$ and then with enhanced enzyme-labeled goat anti-rabbit $\mathrm{IgG}$ polymer (reagent 2 ) at $37^{\circ} \mathrm{C}$ for $30 \mathrm{~min}$. The sections were then developed using 3,3'-diaminobenzidine (DAB) (ZSGB-Bio; OriGene Technologies, Inc.), followed by counterstaining with hematoxylin (ZSGB-Bio; OriGene Technologies, Inc.). Positively stained cells in five random views from three sections per mouse were visualized using a DP71 light microscope with DP controller 3.3.1.292 software (Olympus Corporation) and quantified using ImageJ $1.48 \mathrm{v}$ software (National Institutes of Health).

Total RNA extraction and reverse transcription-quantitative $P C R(R T-q P C R)$. RNA extraction was performed according to the Minimum Information Published in Quantitative Real-time
PCR Experiments (MIQE) guidelines (25). A tissue extraction kit (cat. no. AP-MN-MS-RNA-250; Axygen; Corning, Inc.) was used to extract total RNA from knee joints of the mice in the 4 and 8 weeks groups. Complementary DNA was synthesized from $1 \mu \mathrm{g}$ total RNA using a TransScript All-in-One First-Strand cDNA Synthesis kit (cat. no. AT341-01; TransGen Biotech Co., Ltd.), according to the manufacturer's protocol and a S1000 Thermal Cycler (Bio-Rad Laboratories, Inc.). qPCR was performed with $10 \mu 12 \mathrm{X}$ ChamQ SYBR qPCR Master Mix (cat. no. Q311-02; Vazyme Biotech Co., Ltd.), $0.4 \mu \mathrm{l}$ primers and $1 \mu \mathrm{l}$ template cDNA (total reaction volume, $20 \mu \mathrm{l})$. The qPCR parameters were as follows: $95^{\circ} \mathrm{C}$ for $30 \mathrm{sec}, 40$ cycles of $95^{\circ} \mathrm{C}$ for $10 \mathrm{sec}$ and $60^{\circ} \mathrm{C}$ for $1 \mathrm{~min}$. qPCR was performed in triplicate using an iQ5 system (Bio-Rad Laboratories, Inc.). The primer sequences used in the present study are listed in Table I.

$\beta$-actin was used as a reference gene. Relative gene expression was calculated using the $2^{-\Delta \Delta C \mathrm{q}}$ method (26).

Micro-CT. The mouse knee joint was scanned with a micro-CT device (SkyScan 1176; Bruker Belgium S.A./N.V.) and NRecon v1.6 software (Bruker), and reconstructed micro-CT images were obtained. Data analysis software (CTAn v1.9; Bruker) and three-dimensional model visualization software (CTVol v2.0; Bruker) were used to analyse the data. Visual evaluation of structure in the images was performed, and the quantitative morphometric index was determined based on microtomographic data obtained from the three-dimensional morphometric measurements (27). The area of interest was between the proximal tibial growth plate and the tibial plateau. Evaluation indicators included the bone volume fraction (BV/TV; \%), bone mineral density (BMD; $\left.\mathrm{g} / \mathrm{cm}^{3}\right)$ and trabecular separation (Tb.Sp; mm).

Statistical analysis. All data are expressed as the mean \pm SD. GraphPad Prism 8 software (Graphpad Software, Inc.) was used for the statistical analysis. One-way ANOVA followed by Tukey's multiple comparison test was used to compare data among groups after testing the data for homogeneity of variance. Non-parametric data (OARSI scores) were analysed using the Kruskal-Wallis $\mathrm{H}$ test followed by Dunn's test. $\mathrm{P}<0.05$ was considered to indicate a statistically significant difference.

\section{Results}

Metformin can decrease cartilage degradation in a DMM model. To study the effect of metformin treatment on cartilage degradation in the DMM-induced OA model, histological 
Table I. Primer sequences used in reverse transcription-quantitative PCR.

\begin{tabular}{lll}
\hline Gene & \multicolumn{1}{c}{ Forward primer $\left(5^{\prime}-3^{\prime}\right)$} & Reverse primer $\left(5^{\prime}-3^{\prime}\right)$ \\
\hline NLRP3 & TGCCGTGGTCTCTTCTCAAG & GTCGAAGCAGCATTGATGGG \\
Caspase-1 & TGATGGCATTAAGAAGGCCCA & TCCAAGTCACAAGACCAGGC \\
GSDMD & ATGGGAACATTCAGGGCAGAG & ACCTCAGTGATCTGCACTTCC \\
IL-1 $\beta$ & TGACGGACCCCAAAAGATGAAG & AGCTCTTGTTGATGTGCTGC \\
$\beta$-actin & GTGCTATGTTGCTCTAGACTTCG & ATGCCACAGGATTCCATACC
\end{tabular}

NLRP3, NOD-like receptor protein 3; GSDMD, gasdermin D.
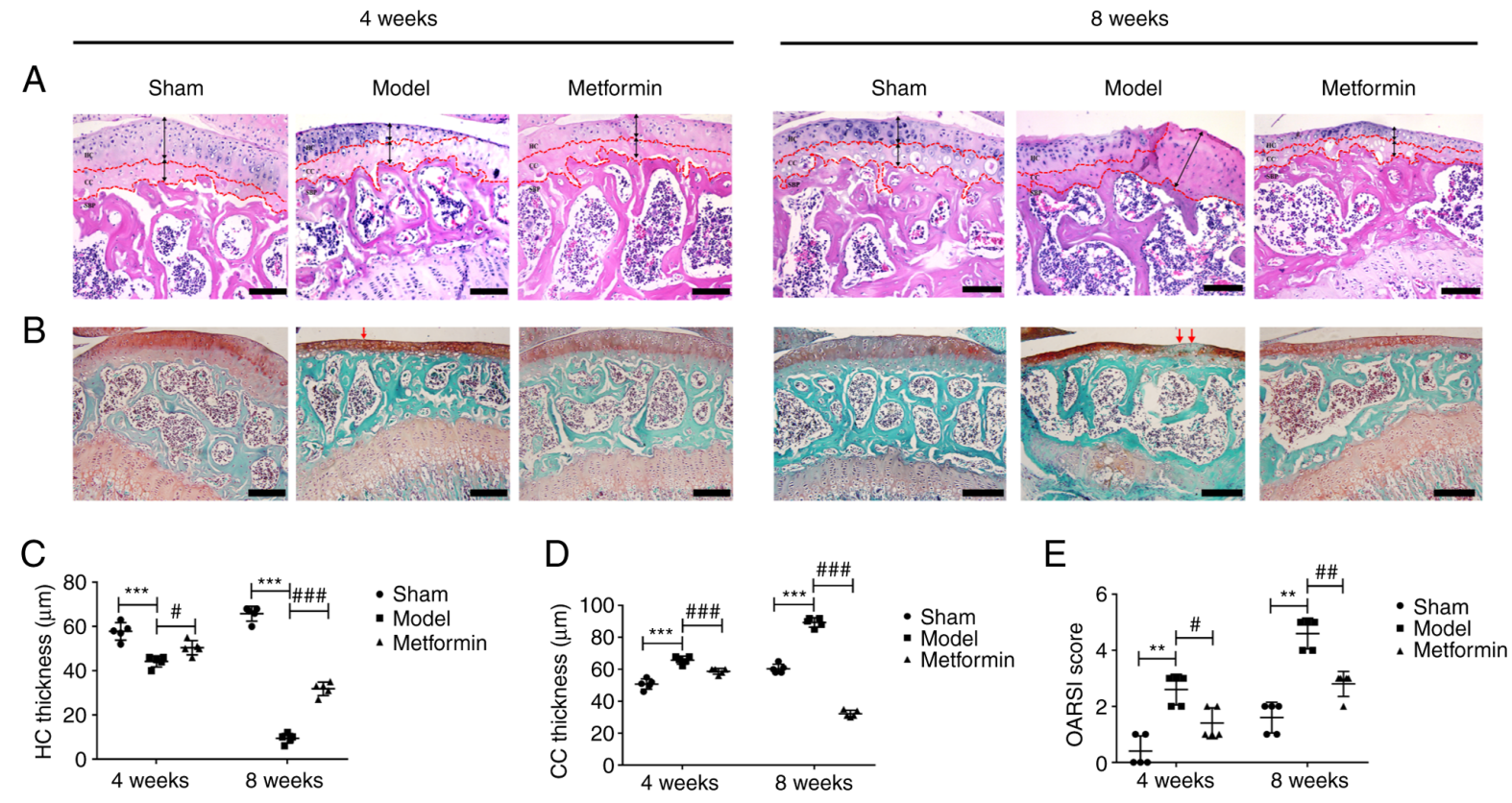

Figure 2. Metformin can decrease cartilage degradation in a destabilization of the medial meniscus model. (A) Mouse H\&E staining results (magnification, $\mathrm{x} 100$ ). $\mathrm{HC}$ and $\mathrm{CC}$ thickness are marked by dotted lines. Black arrows indicated the variation in $\mathrm{HC}$ and $\mathrm{CC}$ thickness. Scale bar=200 $\mu \mathrm{m}$. (B) Mouse safranin O-fast green staining results (magnification, x100). Red arrows indicated the cartilage degradation. Scale bar=200 $\mu \mathrm{m}$. (C) HC and (D) CC thickness. (E) OARSI scores of cartilage structural damage in mice. One-way ANOVA followed by Tukey's multiple comparison test was used to compare data among groups after testing the data for homogeneity of variance. Non-parametric data (OARSI scores) were analysed using the Kruskal-Wallis H test followed by Dunn's test. ${ }^{* *} \mathrm{P}<0.01 ;{ }^{* * *} \mathrm{P}<0.001 ;{ }^{\#} \mathrm{P}<0.05 ;{ }^{\# \#} \mathrm{P}<0.01$; ${ }^{\# \# \#} \mathrm{P}<0.001$. HC, hyaline cartilage; CC, calcified cartilage; OARSI, Osteoarthritis Research Society International.

analysis of safranin O-fast green staining was performed 4 and 8 weeks after DMM surgery. In the sham operation group, the articular cartilage surface was complete and smoothly connected, the tide line was obvious and clear, the cartilage thickness was moderate, the number of cartilage cells was normal, and the structure was regular. Compared with that of the sham operation group, the cartilage of the DMM group exhibited surface destruction, discontinuity, a decreased number of cells and a large amount of proteoglycan loss. At 8 weeks after DMM surgery, HC was lost and CC was evident. Metformin gavage treatment significantly increased the thickness of the articular cartilage and decreased cartilage damage compared with that in the DMM group (Fig. 2A and B). The OARSI histology scoring system was used to evaluate the safranin O-fast green-stained sections. The OARSI score of the DMM group was significantly higher than that of the sham group (Fig. 2E). At 4 weeks after DMM surgery, the metformin treatment group showed a lower OARSI score compared with the DMM group. At 8 weeks after DMM surgery, a significant decrease in the OARSI score was observed in the metformin-treated mice compared with that in the DMM-only mice. It was also demonstrated that DMM surgery resulted in a significant decrease in $\mathrm{HC}$ thickness and a significant increase in CC thickness, and that treatment with metformin was able to reverse these changes in mice at 4 and 8 weeks after DMM surgery (Fig. 2C and D).

Metformin can improve subchondral bone remodelling in a DMM model. To evaluate the structural changes in subchondral bone in the metformin-treated OA model mice, three-dimensional imaging was performed using micro-CT, and quantitative morphometric indicators were analysed. Metformin prominently decreased DMM-induced osteophyte formation and destruction of articular cartilage (Fig. 3A). At 4 weeks, compared with the sham group, the DMM group demonstrated a pathological change, exhibiting subchondral 
4 weeks

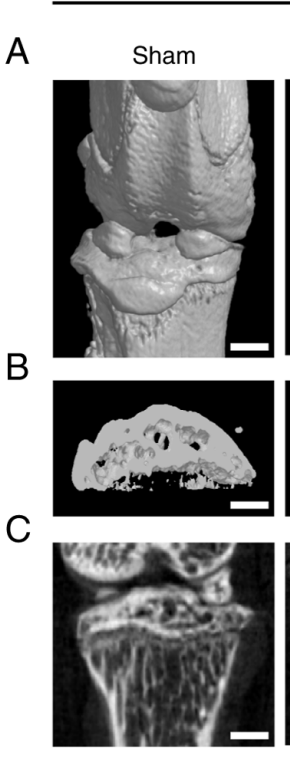

D

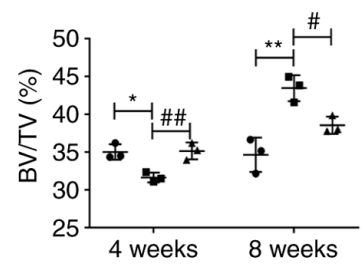

Model
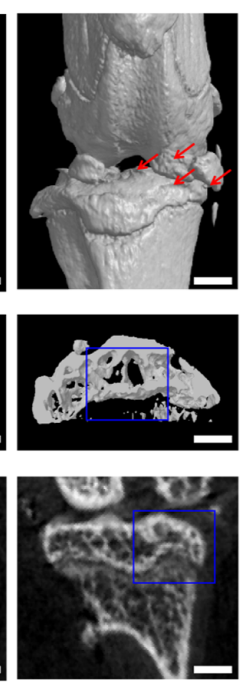

E
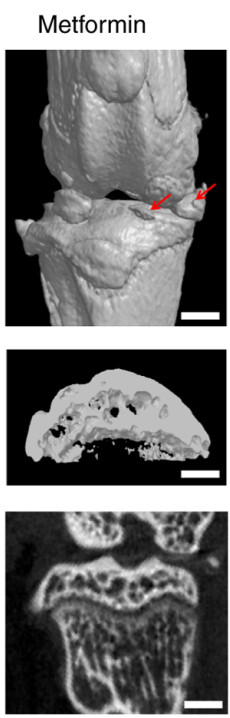
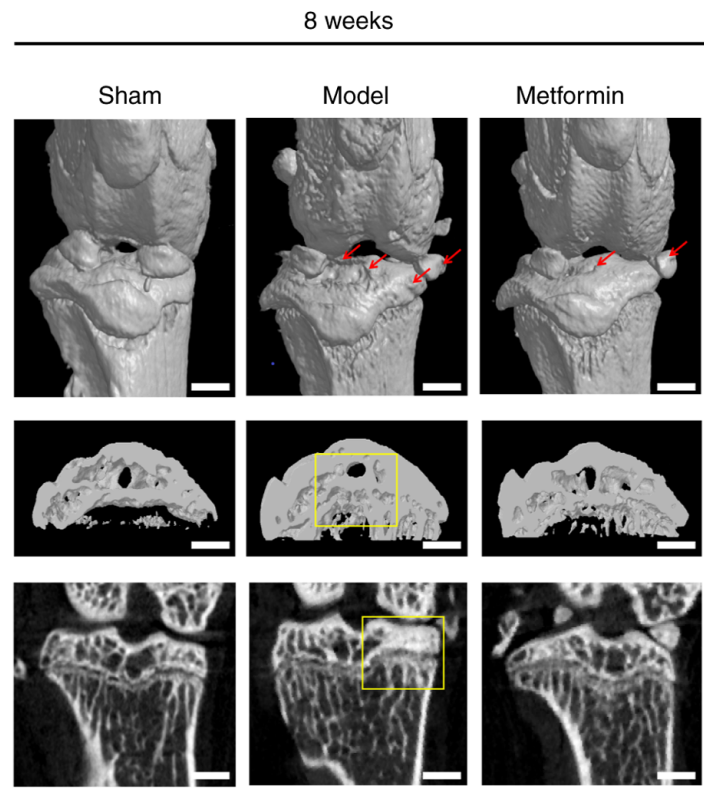

$\mathrm{F}$
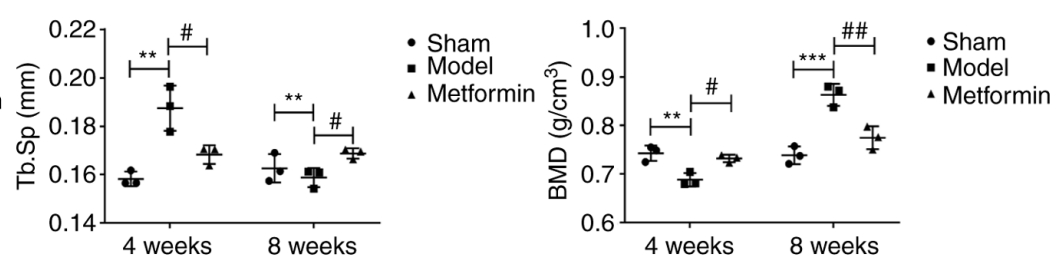

Figure 3. Metformin can improve subchondral bone remodelling in a destabilization of the medial meniscus model. (A) Representative micro-CT images. Osteophyte and cartilage degeneration are marked by red arrows. Scale bar=2,000 $\mu \mathrm{m}$. (B) Micro-CT two-dimensional scan of tibial subchondral bone. Scale bar=2,000 $\mu \mathrm{m}$. (C) Micro-CT two-dimensional reconstructions of tibial subchondral bone. Scale bar=2,000 $\mu \mathrm{m}$. Subchondral bone loss in the tibia is highlighted using a blue box. Increases in bone mass and bone sclerosis are highlighted using a yellow box. Quantification of bone morphological parameters (D) BV/TV, (E) Tb.Sp and (F) BMD. One-way ANOVA followed by Tukey's multiple comparison test was used to compare data among groups after testing the data for homogeneity of variance. ${ }^{*} \mathrm{P}<0.05 ;{ }^{* *} \mathrm{P}<0.01$ and ${ }^{* * *} \mathrm{P}<0.001 ;{ }^{\#} \mathrm{P}<0.05 ;{ }^{\# \#} \mathrm{P}<0.01$. BV/TV, bone volume fraction; Tb.Sp, trabecular separation; BMD, bone mineral density.

bone loss in the tibia. At 8 weeks, a notable increase in bone mass was observed in the DMM group compared with that in the sham group, and bone sclerosis was present in the DMM mice. In the metformin treatment group, expansion of the bone marrow cavity and loss of bone mass were not observed at 4 weeks. At 8 weeks, there was also no increase in either bone mass or bone sclerosis in the metformin treatment group compared with that in the DMM group (Fig. 3B and C). Furthermore, at 4 weeks, BV/TV and BMD in the DMM group were decreased compared with those values in the sham group, while Tb.Sp was increased in the DMM group compared with that in the sham group. These changes were partially reversed in the metformin treatment group. At 8 weeks, BV/TV and BMD were significantly increased and Tb.Sp was decreased in the DMM group compared with those values in the sham group, while in the metformin treatment group, these changes were significantly mitigated (Fig. 3D-F). The changes in the aforementioned parameters indicated that metformin could improve subchondral bone remodelling in DMM model mice.

\section{Metformin can enhance cartilage matrix anabolism and inhibit} its catabolism. To explore the mechanism by which metformin delays cartilage damage in the mouse OA model, immunohistochemical staining of Col II and MMP-13 was performed, as presented in Fig. 4. The results of immunohistochemical staining demonstrated that the expression of MMP-13 was significantly increased after 4 and 8 weeks. Aberrantly expressed MMP-13 was recovered in the metformin treatment group compared with the DMM group at both 4 and 8 weeks following surgery (Fig. 4A and C). In addition, the expression of Col II was significantly decreased after 4 and 8 weeks. Aberrantly expressed Col II was recovered in the metformin treatment group compared with the DMM group at both 4 and 8 weeks following surgery (Fig. 4B and D). In summary, metformin had a protective effect in the DMM-induced OA model, which was mainly mediated through the upregulation of Col II expression and the downregulation of MMP-13 expression.

Metformin can decrease chondrocyte pyroptosis in a DMM model. To investigate whether cartilage degradation is related to chondrocyte pyrolysis, NLRP3, caspase-1, GSDMD and IL-1 $\beta$ immunohistochemical staining and RT-qPCR detection was performed on mouse knee joints collected at 4 and 8 weeks after DMM. The results of immunohistochemical staining demonstrated that the expression of NLRP3, caspase-1, GSDMD and IL-1 $\beta$ were significantly increased after 4 and 8 weeks. Aberrantly expressed NLRP3, caspase-1, GSDMD and IL-1 $\beta$ were recovered in the metformin treatment group compared with the DMM group at both 4 and 8 weeks following 

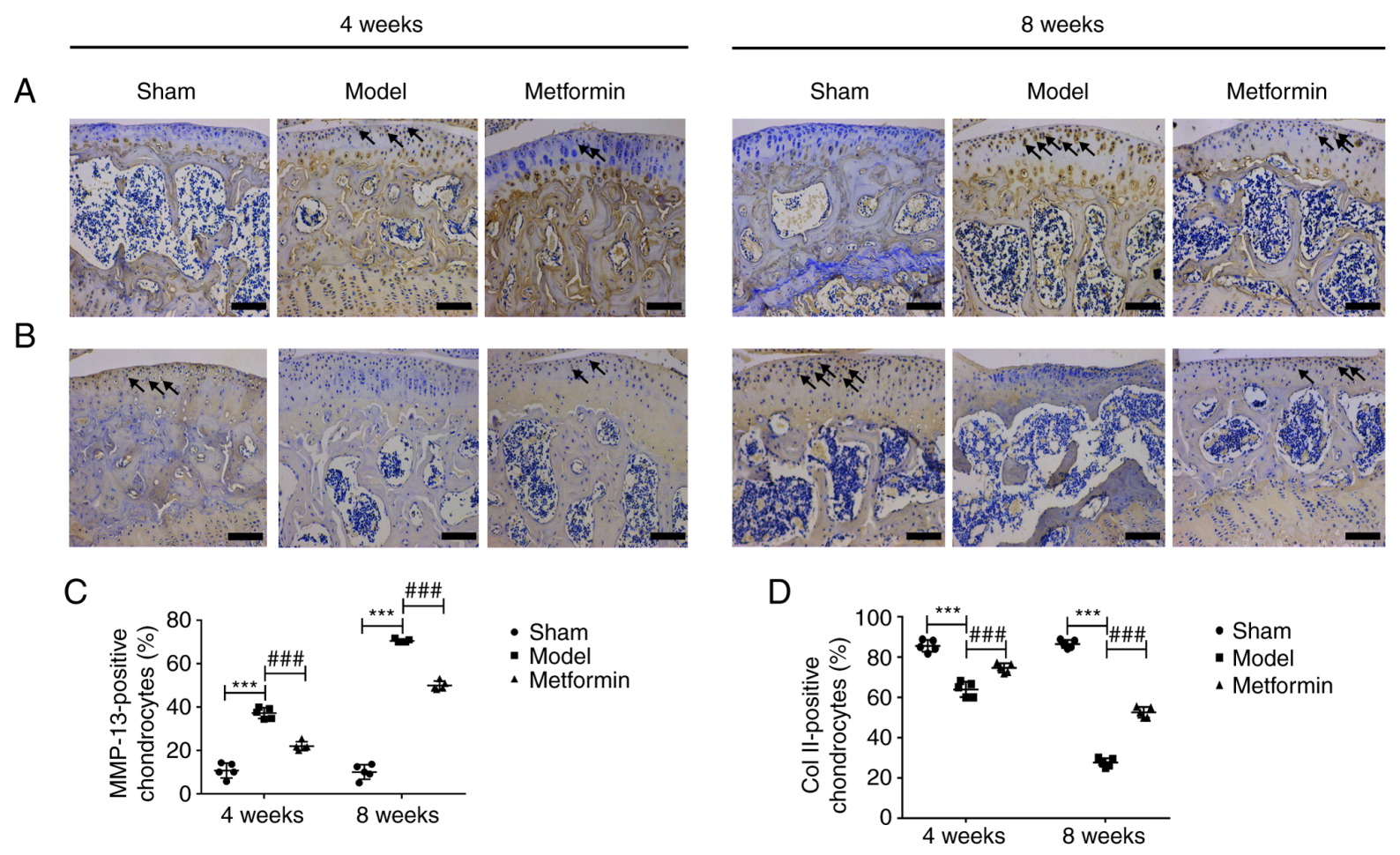

Figure 4. Metformin can enhance cartilage matrix anabolism and inhibit its catabolism. (A) MMP-13 and (B) Col II immunohistochemistry test results (magnification, $\mathrm{x} 400$ ). Scale bar $=100 \mu \mathrm{m}$. Black arrows indicate the positive cells. The ratios of positive cells immunoreactive for (C) MMP-13 and (D) Col II. One-way ANOVA followed by Tukey's multiple comparison test was used to compare data among groups after testing the data for homogeneity of variance. ${ }^{* * * *} \mathrm{P}<0.001 ;{ }^{\# \# *} \mathrm{P}<0.001$. MMP-13, matrix metalloproteinase 13; Col II, type II collagen.

surgery (Fig. 5). The RT-qPCR results were consistent with those from immunohistochemical staining. The mRNA levels of NLRP3, caspase-1, GSDMD and IL- $1 \beta$ were significantly increased after 4 and 8 weeks. The abnormal mRNA levels of NLRP3, caspase-1, GSDMD and IL-1 $\beta$ were recovered in the metformin treatment group compared with the DMM group at both 4 and 8 weeks following surgery (Fig. 6). These results revealed that treatment with metformin can reduce the level of pyroptosis in OA chondrocytes.

\section{Discussion}

To the best of the authors' knowledge, the present study provides the first demonstration that metformin delays the degeneration of articular cartilage after DMM by inhibiting the activation of the NLRP3 inflammasome. The current study found that metformin can decrease the occurrence of chondrocyte pyroptosis by affecting the activation of NLRP3 inflammasome, and that it can ultimately delay the progression of OA. Knee OA was induced in C57BL/6 mice through DMM. Articular cartilage exhibited early degenerative changes 4 weeks after DMM and late degenerative changes after 8 weeks. The current study demonstrated that metformin not only exerted a chondroprotective effect by increasing $\mathrm{HC}$ thickness and decreasing $\mathrm{CC}$ thickness, cartilage degeneration (as measured using the OARSI score) and osteophyte formation, but also that it enhanced cartilage matrix anabolism, inhibited cartilage catabolism and decreased the occurrence of chondrocyte pyroptosis in articular cartilage.

Pyroptosis is increasingly being identified as closely related to the pathophysiological changes in certain chronic diseases including rheumatoid arthritis and osteoarthritis (28).
Activation of the NLRP3 inflammasome is the core driver of pyroptosis (29). To date, the occurrence of pyrolysis mediated by NLRP3 inflammasome activation in knee OA and the specific mechanisms involved have remained unclear. Denoble et al (30) demonstrated that uric acid in the synovium of patients with OA could activate pre-IL-18 and pre-IL-1 $\beta$ by activating the NLRP 3 inflammasome, and that IL-18 and IL-1 $\beta$ levels in the synovium were positively associated with the severity of OA. Therefore, we hypothesize that pyroptosis mediated by NLRP 3 inflammasome activation may be involved in the process of OA. Our study revealed that in the early stage of OA (4 weeks), cartilage degradation and cartilage matrix catabolism had started to significantly increase compared with the sham group, and anabolic metabolism had started to significantly decrease compared with the sham group. During the same period, chondrocyte pyrolysis was significantly increased. When OA developed to the late stage (8 weeks), cartilage degradation and cartilage matrix catabolism had increased further, and anabolic metabolism had decreased further. During the same period, chondrocyte pyroptosis was also significantly increased. Treatment with metformin reversed the aforementioned changes. The present results indicated that cell pyroptosis is involved in the development of OA and that the degradation of OA chondrocytes may be achieved through chondrocyte pyrolysis. The activation of the NLRP3 inflammasome plays a key role in this process. According to a previous report, the expression of NLRP3, IL-18 and IL-1 $\beta$ in the synovial fluid of mice is markedly increased in mice with collagen-induced arthritis, furthermore, the expression of NLRP3 is associated with the severity of knee OA (31). These observations are consistent with the present study results. 


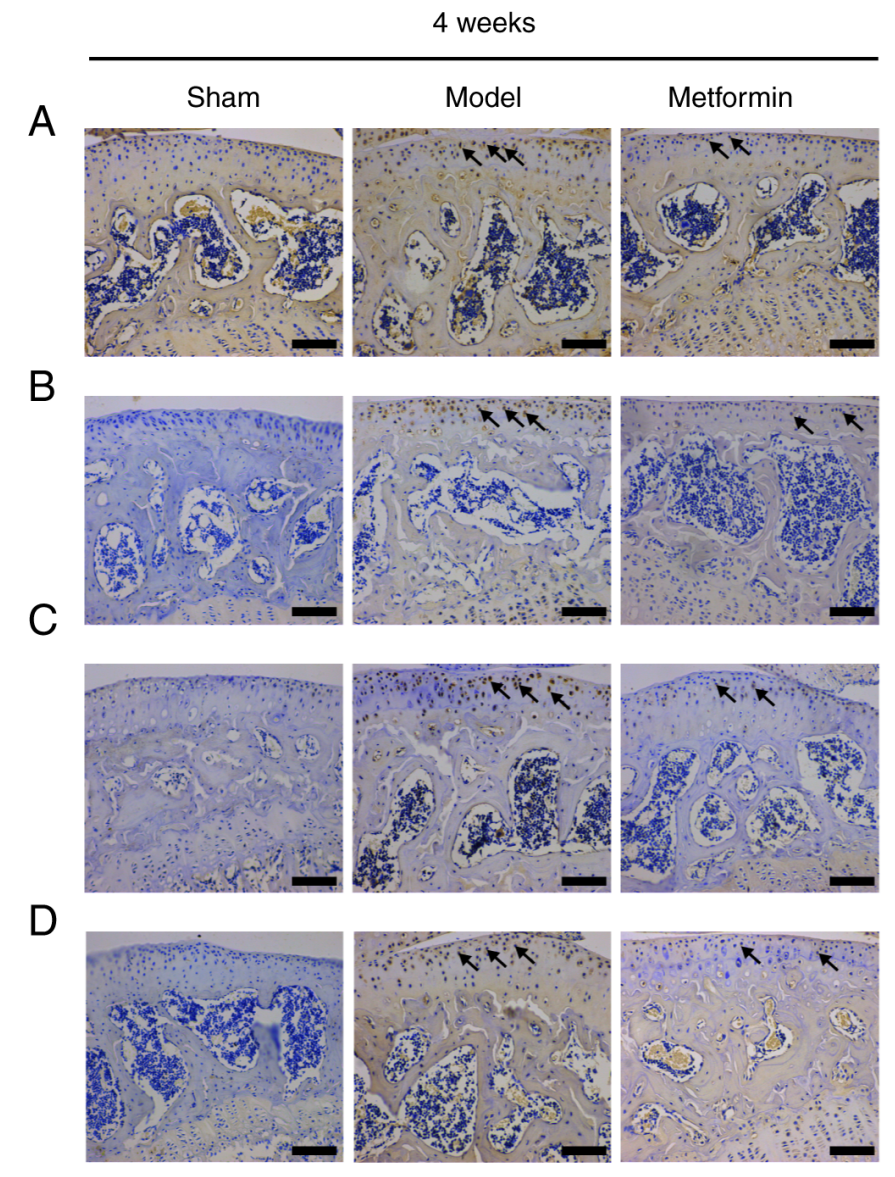

$\mathrm{E}$

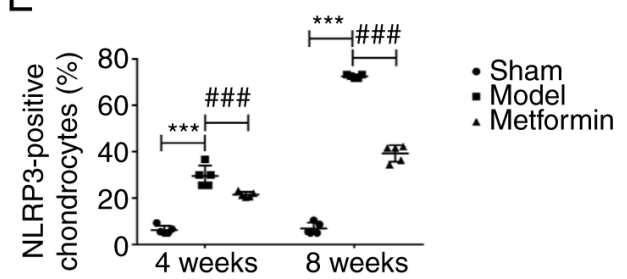

8 weeks
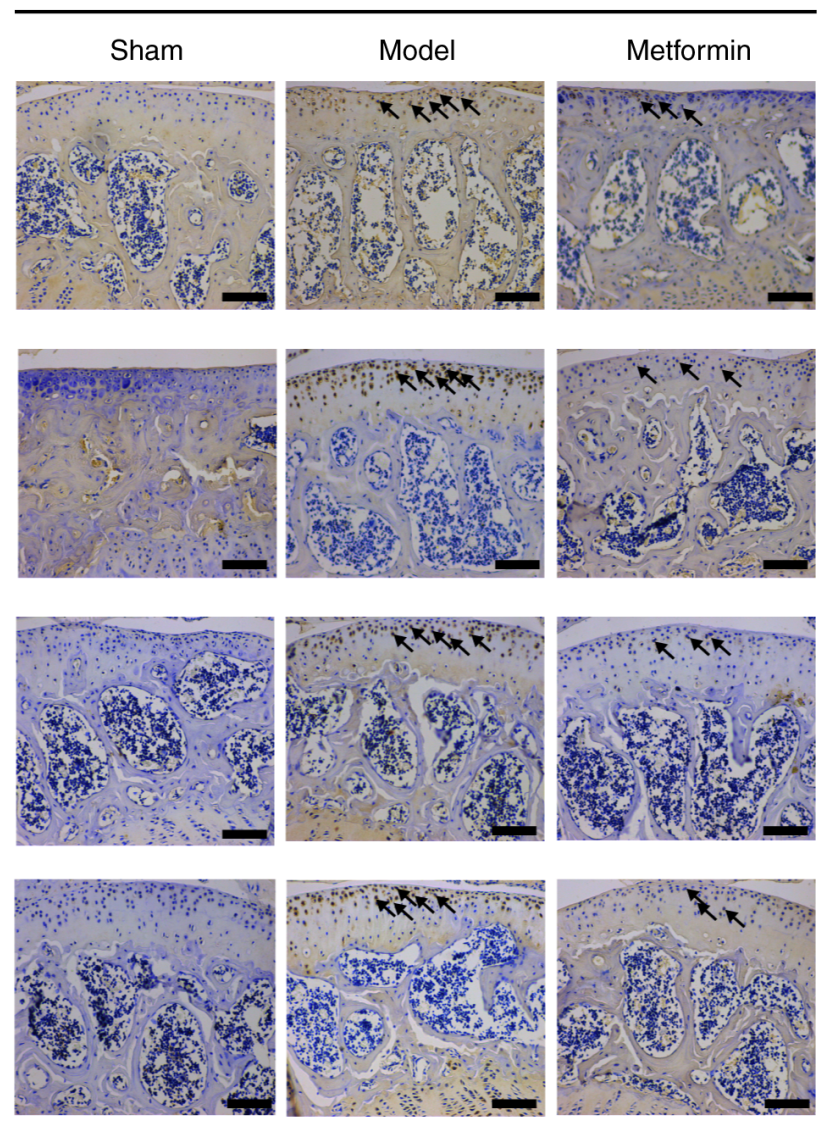

$\mathrm{F}$

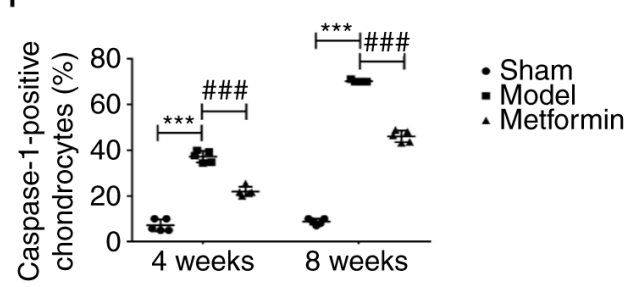

$\mathrm{H}$
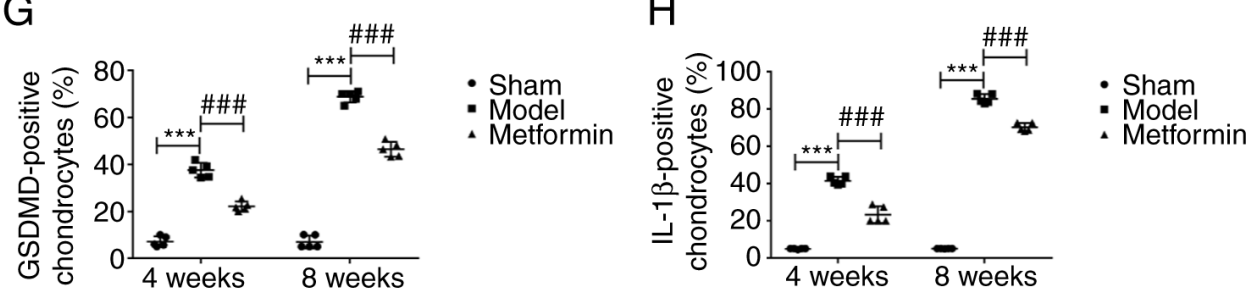

Figure 5. Metformin can decrease chondrocyte pyroptosis in a destabilization of the medial meniscus model at the protein level. (A) NLRP3, (B) caspase-1, (C) GSDMD and (D) IL-1 $\beta$ immunohistochemistry test results (magnification, $x 400$ ). Scale bar $=100 \mu \mathrm{m}$. Black arrows indicate the positive cells. The ratio of positive cells immunoreactive for (E) NLRP3, (F) caspase-1, (G) GSDMD and (H) IL-1ß. One-way ANOVA followed by Tukey's multiple comparison test was used to compare data among groups after testing the data for homogeneity of variance. ${ }^{* * * *} \mathrm{P}<0.001$; ${ }^{\# \# \#} \mathrm{P}<0.001$. NLRP3, NOD-like receptor protein 3 ; GSDMD, gasdermin D.

Xyloside can decrease the synovitis and fibrosis in knee OA by inhibiting HIF-1 $\alpha$ and the NLRP3 inflammasome (32). This observation confirms our study finding that inhibiting chondrocytes pyrolysis may be a feasible strategy for alleviating cartilage degeneration in knee OA. Anti-inflammatory therapy targeting the NLRP3 inflammasome, as an important participant in pyroptosis, may be a novel method for the treatment of OA (33).
Metformin was first used as a hypoglycaemic drug, and it has an anti-inflammatory effect and a regulatory effect on bone (28). Metformin may serve a role in the treatment of OA. Numerous animal studies have shown that metformin affects the development of OA by activating the AMPK signalling pathway $(23,34)$. At the cellular level, metformin protects chondrocytes from IL-1 $\beta$-induced damage by regulating the AMPK/NF- $\kappa B$ signalling pathway (35). These observations 
A

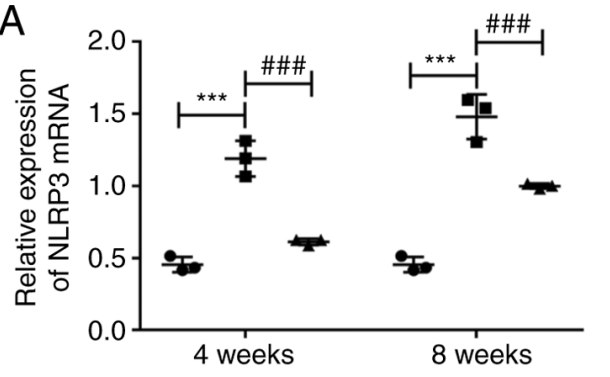

C

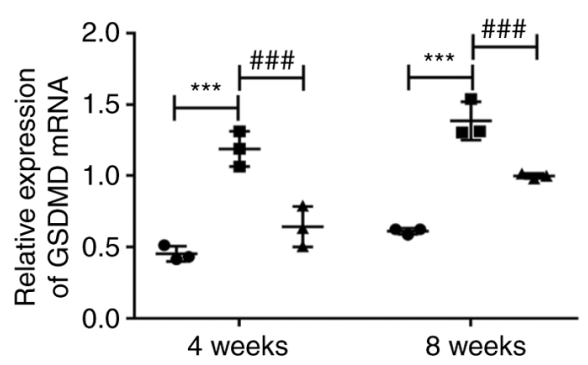

- Sham

- Model

- Metformin
B

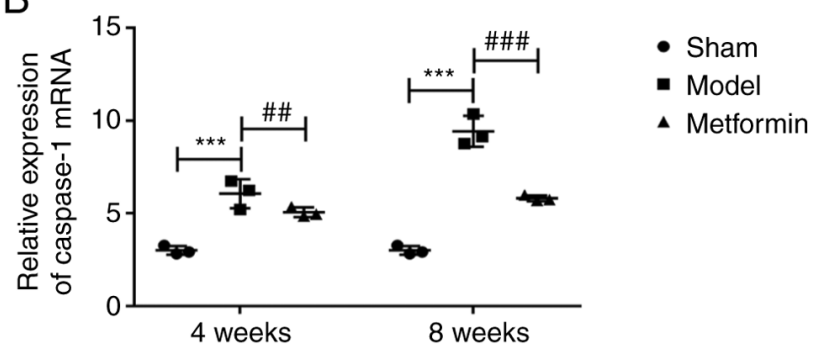

D

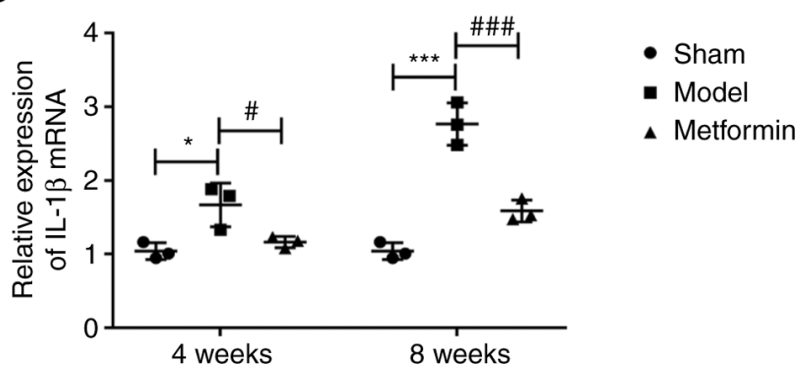

Figure 6. Metformin can decrease chondrocyte pyroptosis in a destabilization of the medial meniscus model at gene level. Relative expression levels of (A) NLRP3, (B) caspase-1, (C) GSDMD and (D) IL-1 $\beta$ mRNA. One-way ANOVA followed by Tukey's multiple comparison test was used to compare data among groups after testing the data for homogeneity of variance. ${ }^{*} \mathrm{P}<0.05$ and ${ }^{* * * *} \mathrm{P}<0.001 ;{ }^{\#} \mathrm{P}<0.05 ;{ }^{\# \#} \mathrm{P}<0.01 ;{ }^{\# \#} \mathrm{P}<0.001$. NLRP3, NOD-like receptor protein 3; GSDMD, gasdermin D.

are consistent with our study findings. Metformin treatment decreased cartilage degradation, inhibited cartilage matrix catabolism and enhanced cartilage matrix anabolism in both the early and late stages of OA in our study. Immunohistochemistry and RT-qPCR analyses were employed, and indicated that metformin effectively inhibited the occurrence of chondrocyte pyrolysis in OA mice. Previous studies have confirmed that the inhibition of mitochondrial ATP and DNA synthesis by metformin inhibits the activation of NLRP3 inflammasome and lung inflammation (36), and can improve depression-like symptoms in mice by inhibiting peripheral and central NF- $\mathrm{B}$-NLRP3 inflammation activation (37). Based on the aforementioned findings, we hypothesize that metformin can decrease the occurrence of OA chondrocyte pyrolysis by inhibiting the NLRP3 inflammasome, inhibiting cartilage degradation, promoting cartilage anabolism and inhibiting cartilage catabolism. This route may be another important pathway by which metformin delays the progression of OA, in addition to the AMPK pathway. The most important limitation of our study is that the research was limited to the level of small animals. The role of metformin in the development of knee OA through the inhibition of chondrocyte pyroptosis requires further investigation at the cellular level. Regarding the therapeutic effect of metformin in the development of OA, further research is needed, including clinical studies with large cohorts.

Subchondral bone is one of the basic units that constitutes the structure and function of joints by maintaining the normal structure and function of cartilage (38). A number of animal experiments have demonstrated that early damage to the subchondral bone occurs before cartilage degeneration and osteophyte formation, mainly via enhanced bone resorption, which manifests as a decrease in bone volume and a thickening of the CC layer. Later damage is mainly caused by bone formation, manifested as subchondral bone sclerosis $(39,40)$. The present study revealed that metformin could improve this abnormal bone remodelling of the subchondral bone in a DMM model. Bone and cartilage are the functional and structural units of the knee joint, and the present results confirmed that metformin could directly act on chondrocytes and decrease the pyroptosis of OA chondrocytes. It is hypothesized that metformin may also act first on the OA subchondral bone to improve abnormal bone remodelling and indirectly act on chondrocytes through the subchondral bone. Research on the mechanism underlying the effects of metformin on OA cartilage and subchondral bone is required at the animal and cellular levels to confirm this.

In conclusion, the present study demonstrated that metformin improved the progression of OA in a mouse model of DMM surgery-induced OA. Metformin inhibited the activation of NLRP3 inflammasome, decreased cartilage degradation, improved subchondral bone remodelling and inhibited chondrocyte pyroptosis. These findings enhance our understanding of the role of metformin as a promising drug for the treatment of $\mathrm{OA}$.

\section{Acknowledgements}

The authors would like to thank Dr Xueyu Hu (The General Hospital of Ningxia Medical University, Yinchuan, China) for providing experimental technical support.

\section{Funding}

The present study was supported by the Scientific Research Project from Ningxia Province (grant no. 2021AAC03396) and the Scientific Research Project of the Key Research 
and Development Project from Ningxia Province (grant no. 2018BEG02005).

\section{Availability of data and materials}

The datasets used and/or analysed during the current study are available from the corresponding author on reasonable request.

\section{Authors' contributions}

QJ and ZL designed the experiment. JY, DD and GF conducted the experiment. YZ, YY, LM and HG analysed the data, and drafted and revised the manuscript. QJ, ZL and JY confirm the authenticity of all the raw data. All authors read and approved the final manuscript.

\section{Ethics approval and consent to participate}

All experiments were approved by the Animal Experiment Ethics Committee of Ningxia Medical University (Yinchuan, China; protocol no. 2020-115). All experiments were performed under the standard ethical principles of animal experiments.

\section{Patient consent for publication}

Not applicable.

\section{Competing interests}

The authors declare that they have no competing interests.

\section{References}

1. Li Z, Huang Z and Bai L: The P2X7 receptor in osteoarthritis. Front Cell Dev Biol 9: 628330, 2021.

2. Yu D, Jordan KP, Bedson J, Englund M, Blyth F, Turkiewicz A, Prieto-Alhambra D and Peat G: Population trends in the incidence and initial management of osteoarthritis: Age-period-cohort analysis of the clinical practice research datalink, 1992-2013. Rheumatology (Oxford) 56: 1902-1917, 2017.

3. Fu C, Zheng C, Lin J, Ye J, Mei Y, Pan C, Wu G, Li X, Ye H and Liu X: Cibotium barometz polysaccharides stimulate chondrocyte proliferation in vitro by promoting G1/S cell cycle transition. Mol Med Rep 15: 3027-3034, 2017.

4. Fischer H, Koenig U, Eckhart L and Tschachler E: Human caspase 12 has acquired deleterious mutations. Biochem Biophys Res Commun 293: 722-726, 2002.

5. Charlier E, Relic B, Deroyer C, Malaise O, Neuville S, Collée J, Malaise MG and De Seny D: Insights on molecular mechanisms of chondrocytes death in osteoarthritis. Int J Mol Sci 17: 2146, 2016.

6. Liao L, Zhang S, Gu J, Takarada T, Yoneda Y, Huang J, Zhao L, Oh CD, Li J, Wang B, et al: Deletion of Runx2 in articular chondrocytes decelerates the progression of DMM-induced osteoarthritis in adult mice. Sci Rep 7: 2371, 2017.

7. Chen D, Shen J, Zhao W, Wang T, Han L, Hamilton JL and Im HJ: Osteoarthritis: Toward a comprehensive understanding of pathological mechanism. Bone Res 5: 16044, 2017.

8. Wang K, Li Y, Han R, Cai G, He C, Wang G and Jia D: T140 blocks the SDF-1/CXCR4 signaling pathway and prevents cartilage degeneration in an osteoarthritis disease model. PLoS One 12: e176048, 2017.

9. Samways DS, Li Z and Egan TM: Principles and properties of ion flow in P2X receptors. Front Cell Neurosci 8: 6, 2014.

10. Shao BZ, Xu ZQ, Han BZ, Su DF and Liu C: NLRP3 inflammasome and its inhibitors: A review. Front Pharmacol 6: 262, 2015.

11. Swanson KV, Deng M and Ting JPY: The NLRP3 inflammasome: Molecular activation and regulation to therapeutics. Nat Rev Immunol 19: 477-489, 2019.
12. Xing Y, Yao X, Li H, Xue G, Guo Q, Yang G, An L, Zhang Y and Meng G: Cutting edge: TRAF6 mediates TLR/IL-1R signaling-induced nontranscriptional priming of the NLRP3 inflammasome. J Immunol 199: 1561-1566, 2017.

13. Scanzello CR and Goldring SR: The role of synovitis in osteoarthritis pathogenesis. Bone 51: 249-257, 2012.

14. Wang S, Mobasheri A, Zhang Y, Wang Y, Dai T and Zhang Z: Exogenous stromal cell-derived factor-1 (SDF-1) suppresses the NLRP3 inflammasome and inhibits pyroptosis in synoviocytes from osteoarthritic joints via activation of the AMPK signaling pathway. Inflammopharmacology 29: 695-704, 2021.

15. McAllister MJ, Chemaly M, Eakin AJ, Gibson DS and McGilligan VE: NLRP3 as a potentially novel biomarker for the management of osteoarthritis. Osteoarthr Cartilage 26: 612-619, 2018.

16. Di Virgilio F: The therapeutic potential of modifying inflammasomes and NOD-like receptors. Pharmacol Rev 65: 872-905, 2013.

17. Pernicova I and Korbonits M: Metformin-mode of action and clinical implications for diabetes and cancer. Nat Rev Endocrinol 10: 143-156, 2014.

18. Gao Y, Li Y, Xue J, Jia Y and Hu J: Effect of the anti-diabetic drug metformin on bone mass in ovariectomized rats. Eur $\mathrm{J}$ Pharmacol 635: 231-236, 2010.

19. Park MJ, Moon SJ, Baek JA, Lee EJ, Jung KA, Kim EK, Kim DS, Lee JH, Kwok SK, Min JK, et al: Metformin augments anti-inflammatory and chondroprotective properties of mesenchymal stem cells in experimental osteoarthritis. J Immunol 203: 127-136, 2019.

20. Nie L, Zhao P, Yue Z, Zhang P, Ji N, Chen Q and Wang Q: Diabetes induces macrophage dysfunction through cytoplasmic dsDNA/AIM2 associated pyroptosis. J Leukoc Biol 110: 497-510, 2021.

21. Zhang J, Huang L, Shi X, Yang L, Hua F, Ma J, Zhu W, Liu X, Xuan R, Shen Y, et al: Metformin protects against myocardial ischemia-reperfusion injury and cell pyroptosis via AMPK/NLRP3 inflammasome pathway. Aging (Albany NY) 12: 24270-24287, 2020.

22. Tan Y, Chen J, Jiang Y, Chen X, Li J, Chen B and Gao J: The anti-periodontitis action of metformin via targeting NLRP3 inflammasome. Arch Oral Biol 114: 104692, 2020.

23. Feng X, Pan J, Li J, Zeng C, Qi W, Shao Y, Liu X, Liu L, Xiao G, Zhang $\mathrm{H}$, et al: Metformin attenuates cartilage degeneration in an experimental osteoarthritis model by regulating AMPK/mTOR. Aging (Albany NY) 12: 1087-1103, 2020.

24. Glasson SS, Chambers MG, Van Den Berg WB and Little CB: The OARSI histopathology initiative-recommendations for histological assessments of osteoarthritis in the mouse. Osteoarthr Cartilage 18 (Suppl 3): S17-S23, 2010.

25. Kirschneck C, Batschkus S, Proff P, Köstler J, Spanier G and Schröder A: Valid gene expression normalization by RT-qPCR in studies on hPDL fibroblasts with focus on orthodontic tooth movement and periodontitis. Sci Rep 7: 14751, 2017.

26. Livak KJ and Schmittgen TD: Analysis of relative gene expression data using real-time quantitative PCR and the 2(-Delta Delta C(T)) method. Methods 25: 402-408, 2001.

27. Hildebrand T, Laib A, Müller R, Dequeker J and Rüegsegger P: Direct three-dimensional morphometric analysis of human cancellous bone: Microstructural data from spine, femur, iliac crest, and calcaneus. J Bone Miner Res 14: 1167-1174, 1999.

28. Spel L and Martinon F: Inflammasomes contributing to inflammation in arthritis. Immunol Rev 294: 48-62, 2020.

29. Toldo S, Mezzaroma E, Buckley LF, Potere N, Di Nisio M, Biondi-Zoccai G, Van Tassell BW and Abbate A: Targeting the NLRP3 inflammasome in cardiovascular diseases. Pharmacol Ther 236: 108053, 2021 (Online ahead of print).

30. Denoble AE, Huffman KM, Stabler TV, Kelly SJ, Hershfield MS, McDaniel GE, Coleman RE and Kraus VB: Uric acid is a danger signal of increasing risk for osteoarthritis through inflammasome activation. Proc Natl Acad Sci USA 108: 2088-2093, 2011.

31. Zhang Y, Zheng Y and Li H: NLRP3 inflammasome plays an important role in the pathogenesis of collagen-induced arthritis. Mediat Inflamm 2016: 9656270, 2016.

32. Zhang L, Li X, Zhang H, Huang Z, Zhang N, Zhang L, Xing R and Wang P: Agnuside alleviates synovitis and fibrosis in knee osteoarthritis through the inhibition of HIF-1 $\alpha$ and NLRP3 inflammasome. Mediat Inflamm 2021: 5534614, 2021. 
33. Wojdasiewicz P, Poniatowski ŁA and Szukiewicz D: The role of inflammatory and anti-inflammatory cytokines in the pathogenesis of osteoarthritis. Mediat Inflamm 2014: 561459, 2014.

34. Li J, Zhang B, Liu WX, Lu K, Pan H, Wang T, Oh CD, Yi D, Huang J, Zhao L, et al: Metformin limits osteoarthritis development and progression through activation of AMPK signalling. Ann Rheum Dis 79: 635-645, 2020.

35. Zhang M, Liu Y, Huan Z, Wang Y and Xu J: Metformin protects chondrocytes against IL-1 $\beta$ induced injury by regulation of the AMPK/NF- $\kappa$ B signaling pathway. Pharmazie 75: 632-636, 2020.

36. Xian H, Liu Y, Rundberg Nilsson A, Gatchalian R, Crother TR, Tourtellotte WG, Zhang Y, Aleman-Muench GR, Lewis G, Chen W, et al: Metformin inhibition of mitochondrial ATP and DNA synthesis abrogates NLRP3 inflammasome activation and pulmonary inflammation. Immunity 54: 1463-1477.e11, 2021.

37. Du RW and Bu WG: Metformin improves depressive-like symptoms in mice via inhibition of peripheral and central NF-KB-NLRP3 inflammation activation. Exp Brain Res 238: 2549-2556, 2020.
38. Muraoka T, Hagino H, Okano T, Enokida M and Teshima R: Role of subchondral bone in osteoarthritis development: A comparative study of two strains of guinea pigs with and without spontaneously occurring osteoarthritis. Arthritis Rheum 56: 3366-3374, 2007.

39. Hayami T, Pickarski M, Zhuo Y, Wesolowski GA, Rodan GA and Duong LT: Characterization of articular cartilage and subchondral bone changes in the rat anterior cruciate ligament transection and meniscectomized models of osteoarthritis. Bone 38: 234-243, 2006.

40. Fang H, Huang L, Welch I, Norley C, Holdsworth DW, Beier F and Cai D: Early changes of articular cartilage and subchondral bone in the DMM mouse model of osteoarthritis. Sci Rep 8: 2855,2018 International (CC BY-NC-ND 4.0) License. 